\title{
IMUNOHEMATOLOGIA VETERINÁRIA: ANTÍGENOS ERITROCITÁRIOS CANINOS
}

\author{
Alexandre Gomes Vizzoni ${ }^{1}$ \\ Miguel Ângelo Silva Medeiros²
}

VIZZONI, A. G.; MEDEIROS, M. A. S. Imunohematologia veterinária: antígenos eritrocitários caninos. Arq. Ciênc. Vet. Zool. UNIPAR, Umuarama, v. 20, n. 4, p. 241-246, out./dez. 2017.

\begin{abstract}
RESUMO: A imunohematologia veterinária vem ganhando interesse nos últimos anos devido a maior acessibilidade a tecnologias de detecção de antígenos e anticorpos, interesse dos donos e médicos veterinários em buscar uma melhor qualidade de vida para os animais e as necessidades de transfusões com o menor índice possível de reações indesejadas. Os cães possuem antígenos presentes na membrana de suas células vermelhas, podendo causar reações durante e após transfusões. Diferentemente de humanos e felinos, cães não possuem anticorpos naturais para os principais antígenos, a priori podendo ser transfundidos com qualquer tipo sanguíneo sem consequências posteriores, porém, se submetidos a uma segunda transfusão, sendo essa de um tipo sanguíneo incompatível e previamente sensibilizados, as chances de ocorrer reações transfusionais graves aumentam drasticamente, ocasionando danos ao animal, podendo levá-lo à morte. Por conta desses riscos se faz necessário uma maior atenção aos tipos sanguíneos desses animais onde 8 sistemas são reconhecidos internacionalmente classificados como sistema DEA, sendo eles DEA 1 e seus subtipos (DEA 1.1; DEA1.2; DEA 1.3); DEA 3; DEA 4; DEA 5; DEA 6; DEA 7 e DEA 8, e recentemente um novo sistema denominado Dal. Não há disponível ainda soros para os sistemas DEA 6 e DEA 8 , tornando a pesquisa sobre esses antígenos dificultosa.
\end{abstract}

PALAVRAS-CHAVE: Antígenos eritrocitários. Cães. DEA.

\section{VETERINARY IMMUNOHEMATOLOGY: CANINE ERYTHROCYTE ANTIGEN}

\begin{abstract}
Veterinary immunohematology is gaining interest in recent years due to greater accessibility to antigen and antibody detection technologies, the interests of pet owners and veterinarians in seeking a better quality of life for animals, and requirement of transfusions with the lowest possible rate of collateral reactions. Dogs have antigens present in the membrane of their red blood cells that can cause reactions during and after transfusions. Unlike humans and cats, dogs do not have natural antibodies to the key antigens, and a priori they can be transfused with any type of blood without any further consequences. However, if they are ever subjected to a second transfusion, if using incompatible blood types and being previously sensitized, the likelihood of having serious transfusion reactions drastically increase, causing damage to the animal, which may even lead it to death. Due to those risks, greater attention is required to the blood type of those animals, which present 8 systems, internationally recognized and classified as the DEA system, namely DEA 1 and its subtypes (DEA 1.1; DEA 1.2; DEA 1.3); DEA 3; DEA 4; DEA 5; DEA 6; DEA 7 and DEA 8, and recently a new system referred to as Dal. No serum is yet available for DEA 6 and DEA 8 systems, hindering the research on those antigens.
\end{abstract}

KEY WORDS: Erythrocyte antigens. Dogs. DEA.

\section{INMUNOHEMATOLOGÍA VETERINARIA: ANTÍGENOS ERITROCITARIOS CANINOS}

RESUMEN: La inmunohematología veterinaria ha ganado atención en los últimos años debido mayor accesibilidad a tecnologías de detección de antígenos y anticuerpos, interés de dueños y médicos veterinarios en buscar mejor calidad de vida para los animales y las necesidades de transfusiones con menor índice posible de reacciones indeseadas. Los perros poseen antígenos presentes en la membrana de sus células rojas, pudiendo causar reacciones durante y después de transfusiones. Diferentemente de humanos y felinos, perros no tienen anticuerpos naturales para los principales antígenos, a priori, pudiendo ser transfundidos con cualquier tipo de sangre sin consecuencias posteriores, todavía, si sometidos a una segunda transfusión, siendo esa de un tipo sanguíneo incompatible y previamente sensibilizados, la posibilidad de ocurrir reacciones transfusional grave aumenta drásticamente, ocasionando daños al animal, pudiendo llevarlo a la muerte. Por esos riesgos se hace necesario más atención a los tipos sanguíneos de esos animales, donde 8 sistemas son reconocidos internacionalmente y clasificados como sistema DEA, siendo ellos DEA 1 y sus subtipos (DEA 1.1; DEA 1.2; DEA 1.3); DEA 3; DEA 4; DEA 5; DEA 6; DEA 7 y DEA 8, y recién un nuevo sistema denominado Dal. No hay aún disponible sueros para los sistemas DEA 6 y DEA 8 , haciendo dificultosa la investigación sobre esos antígenos.

PALABRAS CLAVE: Antígenos eritrocitarios. DEA. Perros.

DOI: 10.25110 /arqvet.v20i4.2017.5682

'BSc PhD Instituto Nacional de Infectologia Evandro Chagas, FIOCRUZ. *Autor Correspondente: alexandre.vizzoni@ini.fiocruz.br, Coordenação de Atividades Diagnósticas Av. Brasil, 4365 Manguinhos - RJ - Brasil, Cep. 21040-900.

${ }^{2}$ DVM PhD Universidade Castelo Branco, UCB. 


\section{Introdução}

Os antígenos de eritrócitos de cães (DEA, do inglês dog erytrocyt antigen) referem-se à sistemas de grupos sanguíneos caninos originalmente definidos por um comitê internacional para imunogenetica canina, e foi baseado em estudos que utilizaram aloanticorpos induzidos (VRIESENDORP et al., 1973).

Estudos iniciais sobre grupos sanguíneos caninos foram relatados em 1910. Desde então, mais de 20 grupos sanguíneos diferentes foram relatados em cães. No entanto, estudos rigorosos não foram realizados para determinar se todos os grupos relatados são sorologicamente distintos (COLLING; SAISON, 1980; HALL, 1970; SYMONS; BELL, 1992). Como os cães não possuem aloanticorpos de ocorrência natural clinicamente significativos, os grupos sanguíneos em cães foram elucidados no laboratório por meio da indução de anticorpos por transfusão incompatível (HOHENHAUS, 2004).

Os cães possuem atualmente oito tipos sanguíneos bem reconhecidos e de importância transfusional, contendo mais de 20 especificidades antigênicas (BLAIS et al., 2007; HALE, 1995; MESA-SANCHEZ et al., 2014). Os sistemas sanguíneos caninos são divididos em DEA 1; (DEA 1.1;
DEA 1,2; DEA 1,3); DEA 3; DEA 4; DEA 5; DEA 6; DEA 7 e DEA 8 e mais recentemente foram descritos o grupo DAL (encontrado em dálmatas) e DAI (EULER et al., 2016; LEE; GIGER; KIM, 2017; MEDINA VALENTIN; GAVAZZA; LUBAS, 2017). No entanto, pouco se sabe sobre muitos desses tipos de sangue e para alguns, como DEA 6 e 8, os soros de tipagem não existem mais (SPADA et al., 2017).

Semelhante a humanos, cães herdam geneticamente o grupo sanguíneo, podendo expressar mais de um antígeno eritrocitário, sem que haja dominância aparente entre eles, porém, não expressam DEA 1.1, DEA 1.2 e DEA 1.3 simultaneamente por serem expressos no mesmo locus gênico. Podendo expressar, por exemplo, o tipo sanguíneo: DEA $1.1 \mathrm{e}$ DEA 5 ou DEA 1.2 e DEA 4, porém, não é possível expressar DEA 1.1 e DEA 1.2 simultaneamente.

Originalmente, os grupos sanguíneos caninos foram identificados com letras do alfabeto (YOUNG et al., 1952). A convenção atual é usar o acrônimo DEA para o antígeno de eritrócitos do cão, seguido da designação numérica do grupo sanguíneo (Tabela 1). Diferentes grupos de sangue canino receberam padronização internacional, mas ainda hoje, os soros de determinação estão disponíveis quase que exclusivamente apenas para cinco desses grupos.

Tabela 1: Nomenclatura e incidência de grupos sanguíneos caninos

\begin{tabular}{c|c|c|c}
\hline Nomenclatura original & Nomenclatura modificada & Nomenclatura atual & Incidência na população** $^{*}$ \\
\hline CEA 1 & A & DEA 1.1 & $23,4 \%$ (Austrália); 72,7\% (Japão) \\
\hline CEA 2 & A2 2 DEA 1.2 & $4 \%$ (Holanda); 42\% (São Paulo) \\
\hline & & DEA $1.3^{*}$ & Desconhecido \\
\hline CEA 3 & B & DEA 3 & $5 \%$ (Holanda); 24\% (Japão) \\
\hline CEA 4 & C & DEA 4 & $56 \%$ (Holanda); 98,4\% (EUA) \\
\hline CEA 5 & D & DEA 5 & $8 \%$ (Holanda); 98,4\% (EUA) \\
\hline CEA 6 & F & DEA 6 & $60 \%$ (Japão); 99,4\% (EUA) \\
\hline CEA 7 & Tr & DEA 7 & 8 -82\% (EUA) \\
\hline
\end{tabular}

* Descoberto após a nomenclatura modificada (SYMONS; BELL, 1991).

** (MARQUES, 2010).

Como os cães não parecem ter aloanticorpos que ocorrem naturalmente, esses tipos sanguíneos foram originalmente definidos após a sensibilização clínica experimental ou acidental de cães por transfusões incompatíveis (POLAK et al., 2015).

Até o momento, apenas os sistemas DEA 1 e DEA 7 apresentam múltiplos alelos. O emprego de lectinas de plantas tem sido investigado como ferramentas de determinação sanguínea, mas as reações de aglutinação não diferenciaram os tipos conhecidos de sangue canino (ANDREWS; CHAVEY; SMITH, 1992).

A determinação da tipagem sanguínea previamente as transfusões de sangue em cães, minimiza o risco de uma reação de transfusão devido à incompatibilidade entre os diferentes tipos de sangue existentes (GOY-THOLLOT et al., 2017). Portanto, informações sobre a prevalência destes antígenos eritrocitários em várias raças, ajudam na seleção de doadores de sangue para a tomada de decisão e a criação de um programa de doadores de sangue caninos.

\section{Antígenos eritrocitários caninos}

a) Antígenos DEA 1

DEA 1 é o tipo sanguíneo canino mais estudado. A prevalência de cães de DEA 1-positivos varia tanto geograficamente, que são apresentadas frequências entre 100\% a $<10 \%$, tendo sido estimado em aproximadamente 40-60\% sua prevalência internacional (CARLI et al., 2017; SPADA et al., 2017).

Praticamente, todos os DEAs podem induzir a formação de aloanticorpos, mas o antígeno mais imunogênico parece ser DEA 1.1 (HOHENHAUS, 2004) [2, 4]. Após uma transfusão incompatível com DEA 1 , o receptor de sangue torna-se sensibilizado e uma segunda transfusão incompatível com DEA 1 pode resultar em uma reação transfusional aguda e potencialmente fatal (SPADA et al., 2017; TOCCI, 2010). Historicamente, são apresentados diferentes alelos no DEA 1, sendo DEA 1.1, DEA 1.2 e DEA 1.3. A ausência dos três antígenos mencionados acima indica um cão DEA 1 negativo (CARLI et al., 2017; DAVIDOW, 2013). Recentemente, o sistema do grupo sanguíneo DEA 1 foi descrito 
como um sistema alélico autossômico dominante complexo com níveis variados de expressão do antígeno de superfície (ACIERNO; RAJ; GIGER, 2014; EULER et al., 2016). Então, um cão pode ser classificado como DEA 1 negativo (DEA 1-) ou DEA 1 positivo (DEA 1+) com expressão de antígeno fraco a forte (ACIERNO; RAJ; GIGER, 2014; POLAK et al., 2015).

A informação sobre a frequência de grupos sanguíneos DEA 1+ e DEA 1- entre raças caninas está disponível, embora, em muitos estudos foi abordado um número limitado de raças. Os dados foram reportados no Japão (EJIMA; KUROKAWA; IKEMOTO, 1986), Brasil (ESTEVES et al., 2011; NOVAIS; SANTANA; VICENTIN, 1999), África do Sul (VAN DER MERWE; JACOBSON; PRETORIUS, 2002), EUA (IAZBIK et al., 2010), Portugal (FERREIRA; GOPEGUI; MATOS, 2011), Turquia (ERGUL EKIZ et al., 2011), Espanha (MESA-SANCHEZ et al., 2014), Suíça (RIOND et al., 2011), Romênia (OGNEAN, 2013) e América do Norte (EULER et al., 2016).

\section{b) DEA 3}

Este grupo sanguíneo foi denominado como grupo $\mathrm{B}$ na literatura mais antiga e grupo D1, grupo E, na literatura japonesa. Um estudo japonês descobriu que as raças japonesas nativas, como o Shikoku e Akita, tiveram uma incidência de DEA $3>60 \%$, enquanto que as raças não positivas, como o Setter inglês, Setter maltês e o Beagle, eram predominantemente DEA 3 negativas (EJIMA; NOMURA; BULL, 1994). DEA 3 não foi encontrado em um estudo australiano que testou os antígenos eritrocitários em 122 labradores Retriever (SYMONS; BELL, 1992).

O tipo sanguíneo DEA 3 pode apresentar-se como DEA 3 positivo ou DEA 3 negativo, apresentando, assim, dois fenótipos. DEA 3 positivo é considerado dominante, sendo a ausência desse antígeno considerado como DEA 3 negativo (ESTEVES et al., 2011). Foram descritos anticorpos naturais contra DEA 3, sugerindo o contrário ao que ocorre ao DEA 1, ao qual não ocorrem anticorpos naturais. De acordo com estudos realizados nos Estados Unidos, foram encontrados anticorpos anti-DEA 3 em $20 \%$ da população canina DEA 3 negativa, constando apenas $6 \%$ da população canina dos Estados Unidos como DEA 3 positiva (HALE, 1995).

\section{c) DEA 4}

O sistema DEA 4, apresenta também dois fenótipos, sendo DEA 4 positivo e ausência desse antígeno indicando DEA 4 negativo. A expressão desse antígeno varia de acordo com a raça do cão e localização geográfica, sendo frequente nos Estados Unidos, onde pesquisas sugerem que 98\% da população canina é DEA 4 positiva (HALE, 1995).

Por sua alta frequência na população canina, os cães DEA 4 positivos são considerados doadores universais. Não há casos documentados de anticorpos naturais anti-DEA 4 (KESSLER et al., 2010) como ocorre no caso do DEA 3. Foi sugerido que a transfusão de sangue DEA 4 positivo para paciente DEA 4 negativo não seria maligna e não causaria reação hemolítica (HALE, 1995), porém, foi descrita sensibilização e reação transfusional hemolítica aguda em uma cadela DEA 4 negativa, submetida à múltiplas transfusões de doadores considerados "universais", sendo DEA 4 positivos e negativos para o restante dos antígenos do sistema DEA testados (DEA 1.1, 1.2, 3, 5 e 7) (WARDROP et al., 2005). Segundo estes autores, este quadro indica o desenvolvimento de anticorpos Anti-DEA 4, o que ocasionou a reação hemolítica aguda na cadela descrita. Porém, havendo apenas esse registro de reação hemolítica ocasionado por DEA 4, necessitando assim de mais estudos sobre esse antígeno.

e) DEA 5

Semelhante ao DEA 3 e DEA 4, o sistema DEA 5 possuí o fenótipo dominante DEA 5 positivo, ou ausência do antígeno sendo DEA 5 negativo. Pesquisas descrevem que assim como o DEA 3, foram notificados casos de anticorpos naturais anti-DEA 5 em $10 \%$ da população canina dos Estados Unidos. Segundo Hale (1995), há variações de frequência desse antígeno na população canina, dependendo da raça e localização geográfica dos animais, sendo relativamente baixa nos Estados Unidos.

\section{f) DEA 7}

DEA 7 não é parte integrante da membrana dos eritrócitos caninos. É produzido em outro lugar do corpo em forma solúvel, secretado no plasma e adsorvido na membrana celular. A prevalência relatada de DEA 7 varia entre $6 \%$ e 82\% em várias populações caninas (ESTEVES et al., 2011; SPADA et al., 2016b, 2017).

Os antígenos DEA 7 são descritos entre 40 e $72 \%$ da população canina (ESTEVES et al., 2011; IAZBIK et al., 2010; SPADA et al., 2016a). Entre 10 e 40\% dos cães negativos para esses antígenos têm anticorpos naturais para DEA 7 (SPADA et al., 2016a) e a sobrevivência tardia das hemácias transfundidas em cães com antígenos negativos que recebem sangue DEA 7 positivo deve ser considerada (HALE, 1995).

Os antisoros contra $\operatorname{Tr}$ (DEA 7) reagem de forma cruzada com hemácias humanas de Grupo A; consequentemente, o antígeno $\mathrm{Tr}$ parece ter semelhança com o antígeno $A_{1}$ dos humanos (BOWDLER et al., 1971). A saliva de cães Tr positivo contém atividade anti-A e anti-H 48 Apesar da presença de atividade anti-H na saliva canina, não A substância $\mathrm{H}$ foi identificada em eritrócitos caninos, mas foi identificada em células intestinais caninas (HOHENHAUS, 2004).

\section{g) DEA 6 e DEA 8}

Apesar de reconhecidos no segundo workshop sobre imunogenética canina em 1976, não houve sucesso na reprodução de anticorpos policlonais para os antígenos DEA 6 e DEA 8. Esses antígenos não foram mais estudados, pois sem os kits de tipagem, não há possibilidade de detecção desses antígenos na membrana eritrocitária (HALE, 1995).

\section{Sistema DAL}

Aproximadamente hà uma década, foi descrito um novo tipo de antígeno eritrocitário denominado Dal (BLAIS et al., 2007), descoberto durante a realização de testes de compatibilidade em uma cadela dálmata, transfundida anteriormente e após sendo detectados aloanticorpos de classe IgG, indicando sensibilização da cadela (GOULET et al., 2017). Foram testados sangue de 55 tipos de animais de raças distintas e incluindo doadores das primeiras transfusões, havendo incompatibilidade em todos os 55 casos.

Entre 25 dálmatas testados, 4 apresentaram compa- 
tibilidade com a cadela, excluindo todos os antígenos DEA aos quais existem soro específico para tipificação e levando a crer que se tratava de um tipo diferente de antígeno eritrocitário canino. Técnicas de compatibilidade (crossmatching) foram realizadas, possibilitando também a identificação do novo antígeno, verificando-se também durante este mesmo estudo a frequência comum nos cães da raça dálmata.

No entanto, uma alta prevalência de Dal + foi observada entre cães doadores de sangue, o que sugeriu que Dal poderia ser um antígeno eritrocitário de alta frequência em vez de um sistema de grupo sanguíneo semelhante ao DEA 4 (CALLAN; JONES; GIGER, 1995; GOULET et al., 2017; MELZER et al., 2003). Os cachorros Dal anêmicos provavelmente serão sensibilizados pela primeira transfusão com sangue do tipo Dal + (BLAIS et al., 2007), e se exigir novas transfusões de sangue, os doadores de sangue Dal compatíveis podem ser difíceis de serem encontrados (GOULET et al., 2017).

\section{Terapia transfusional e a importância da determinação dos antígenos eritrocitários}

A terapia transfusional veterinária consiste na transfusão de sangue total ou hemocomponentes de um doador para um receptor, sendo indicada para cães em casos como sangramentos agudos, com o intuito de reparar a oxigenação, prevenir sangramentos e reestabalecer a homeostase; hematócrito muito baixo (menor ou igual a 15\%); hemoglobina menor ou igual a $4 \mathrm{~g} / \mathrm{ml}$; perda de mais de $30 \%$ do volume de sangue total; hipovolemia entre outros fatores que lançam a necessidade de uma transfusão sanguínea (HANN et al., 2014; HOHENHAUS, 2004; JAGODICH; HOLOWAYCHUK, 2016).

Em casos onde existe a real necessidade de transfusão, se faz necessário um teste rápido de compatibilidade, sendo a prova cruzada a mais indicada. O método da prova cruzada é dividido em duas partes: Na primeira etapa ou major crossmatching (considerada a etapa mais importante) mistura-se uma pequena parte do sangue total do doador ou suspensão de hemácias com uma pequena parte do soro do receptor. Nessa etapa ocorre que os anticorpos do receptor (se presentes), reagirão aos antígenos presentes na membrana dos eritrócitos do doador, provocando a aglutinação dos eritrócitos e permitindo a visualização de grumos (KESSLER et al., 2010).

A $2^{\circ}$ etapa ou minor crossmatching, uma pequena parte do sangue total ou suspensão de hemácias do receptor em uma pequena parte do soro do doador, indicando se existe a probabilidade de anticorpos presentes do soro do doador reagirem com antígenos presentes na membrana dos eritrócitos do receptor. Observa-se se haverá formação de grumos, caso contrário, a transfusão poderá ser realizada. Essas provas não identificam o tipo sanguíneo do doador nem do receptor, mas indica se existe alguma incompatibilidade que impossibilite a imediata transfusão.

Em casos de incompatibilidade é de grande importância identificar o tipo sanguíneo do receptor e do doador, para que haja conhecimento sobre qual antígeno acarretou na reação, para que se evite a sensibilização e consequente reação transfusional do receptor, podendo levar à consequências graves e até a morte do animal (ODUNAYO et al., 2017).
Com maior acesso aos soros, a identificação torna-se mais rápida e precisa, tornando a transfusão mais segura e eficaz.

\section{Considerações finais}

Por meio da revisão literária permite-se distinguir que os tipos sanguíneos caninos são variáveis de acordo com a raça e localização geográfica do animal, porém, existindo maior prevalência para o tipo sanguíneo DEA 1.1, sendo este mais imunogênico, ocasionando maiores problemas em casos de incompatibilidade. Em diversos estudos, aponta-se que cães positivos para o sistema DEA 4 e negativos para o restante dos antígenos eritrocitários são considerados doadores universais, visto que a incompatibilidade por DEA 4 é rara e não causa hemólise grave.

Com a evolução da medicina veterinária e um maior interesse de cientistas e veterinários pela área e dos proprietários por seus animais de companhia, os métodos de identificação tornaram-se de grande importância, apesar de ainda ser pouco utilizado no Brasil devido ao alto custo dos soros.

Os estudos apontam para a real necessidade do conhecimento dos antígenos eritrocitários caninos, para que se evitem futuros transtornos e agravantes na saúde do animal. Apontam também para a necessidade da implantação dos testes de tipagem e frequência dos testes de compatibilidade em clínicas veterinárias e bancos de sangue, a fim de ser obter maior agilidade nos tratamentos e minimizar reações adversas, sensibilizações e danos à saúde do animal.

\section{Referências}

ACIERNO, M. M.; RAJ, K.; GIGER, U. DEA 1 expression on dog erythrocytes analyzed by immunochromatographic and flow cytometric techniques. Journal of Veterinary Internal Medicine, v. 28, n. 2, p. 592-598, 2014.

ANDREWS, G. A.; CHAVEY, P. S.; SMITH, J. E. Reactivity of lichen lectins with blood typed canine erythrocytes. Research in Veterinary Science, v. 53, n. 3, p. 315-319, 1992.

BLAIS, M.-C. et al. Canine Dal Blood Type: A red cell antigen lacking in some Dalmatians. Journal of Veterinary Internal Medicine /American College of Veterinary Internal Medicine, v. 21, n. 2, p. 281-286, 2007.

BOWDLER, A. J. et al. Tr: A canine red cell antigen related to the A-antigen of human red cells. Vox Sanguinis, v. 20, n. 6 , p. 542-554, jun. 1971 .

CALLAN, M. B.; JONES, L. T.; GIGER, U. Hemolytic transfusion reactions in a dog with an alloantibody to a common antigen. Journal of Veterinary Internal Medicine, v. 9, n. 4, p. 277-279, ago. 1995.

CARLI, E. et al. Frequency of DEA 1 antigen in 1037 mongrel and PUREBREED dogs in ITALY. BMC

Veterinary Research, v. 13, nov. 2017.

COLLING, D. T.; SAISON, R. Canine blood groups. 1. Description of new erythrocyte specificities. Animal Blood 
Groups and Biochemical Genetics, v. 11, n. 1, p. 1-12, 1980.

DAVIDOW, B. Transfusion medicine in small animals. Veterinary Clinics of North America: Small Animal Practice, v. 43, n. 4, p. 735-756, jul. 2013.

EJIMA, H.; KUROKAWA, K.; IKEMOTO, S. Phenotype and gene frequencies of red blood cell groups in dogs of various breeds reared in Japan. Nihon Juigaku Zasshi. The Japanese Journal of Veterinary Science, v. 48, n. 2, p. 363-368, abr. 1986.

EJIMA, H.; NOMURA, K.; BULL, R. W. Breed differences in the phenotype and gene frequencies in canine $\mathrm{D}$ blood group system. Journal of Veterinary Medical Science, v. 56, n. 4, p. 623-626, ago. 1994.

ERGUL EKIZ, E. et al. Frequency of dog erythrocyte antigen 1.1 in 4 breeds native to different areas in Turkey. Veterinary Clinical Pathology, v. 40, n. 4, p. 518-523, dez. 2011

ESTEVES, V. S. et al. Frequencies of DEA blood types in a purebred canine blood donor population in Porto Alegre, RS, Brazil. Pesquisa Veterinária Brasileira, v. 31, n. 2, p. 178-181, fev. 2011.

EULER, C. C. et al. Survey of two new (Kai 1 and Kai 2) and other blood groups in dogs of North America. Journal of Veterinary Internal Medicine, v. 30, n. 5, p. 1642-1647, set. 2016.

FERREIRA, R. R. F.; GOPEGUI, R. R.; MATOS, A. J. F. Frequency of dog erythrocyte antigen 1.1 expression in dogs from Portugal. Veterinary Clinical Pathology, v. 40, n. 2, p. 198-201, jun. 2011

GOULET, S. et al. Prevalence and mode of inheritance of the Dal blood group in dogs in North America. Journal of Veterinary Internal Medicine, v. 31, n. 3, p. 751-758, maio 2017.

GOY-THOLLOT, I. et al. Pre-and Post-Transfusion alloimmunization in dogs characterized by 2 antiglobulinenhanced cross-match tests. Journal of Veterinary Internal Medicine, v. 31, n. 5, p. 1420-1429, 2017.

HALE, A. S. Canine blood groups and their importance in veterinary transfusion medicine. Veterinary Clinics of North America: Small Animal Practice, v. 25, n. 6, p. 1323-1332, nov. 1995

HALL, D. E. A naturally occurring red-cell antigenantibody system in Beagle dogs. Journal of Small Animal Practice, v. 11, n. 8, p. 543-551, 1 ago. 1970.

HANN, L. et al. Effect of duration of packed red blood cell storage on morbidity and mortality in dogs after transfusion: 3,095 cases (2001-2010). Journal of Veterinary Internal Medicine, v. 28, n. 6, p. 1830-1837, dez. 2014
HOHENHAUS, A. E. Importance of blood groups and blood group antibodies in companion animals. Transfusion Medicine Reviews, v. 18, n. 2, p. 117-126, 1 abr. 2004.

IAZBIK, M. C. et al. Prevalence of dog erythrocyte antigens in retired racing Greyhounds. Veterinary Clinical Pathology, v. 39, n. 4, p. 433-435, dez. 2010.

JAGODICH, T. A.; HOLOWAYCHUK, M. K. Transfusion practice in dogs and cats: an Internet-based survey. Journal of Veterinary Emergency and Critical Care (San Antonio, Tex.: 2001), v. 26, n. 3, p. 360-372, maio 2016

KESSLER, R. J. et al. Dog erythrocyte antigens 1.1, $1.2,3,4,7$, and Dal blood typing and cross-matching by gel column technique. Veterinary Clinical Pathology / American Society for Veterinary Clinical Pathology, v. 39, n. 3, p. 306-316, set. 2010.

LEE, J. H.; GIGER, U.; KIM, H. Y. Kai 1 and Kai 2: Characterization of these dog erythrocyte antigens by monoclonal antibodies. PLoS ONE, v. 12, n. 6, 29 jun. 2017.

MARQUES, C.F.S. Frequência do antigéno eritrocitário DEA 1.1 em canídeos e dos antígenos eritrocitários A, B e AB em felídeos de Lisboa, Portugal, 2010. 98f Dissertação (Mestrado Integrado em Medicina Veterinária) - Faculdade de Medicina Veterinária, Universidade Técnica de Lisboa, Lisboa.

MEDINA VALENTIN, A. A.; GAVAZZA, A.; LUBAS, G. Prevalence of dog erythrocyte antigen 1 in 7,414 dogs in Italy. Veterinary Medicine International, v. 2017, 2017.

MELZER, K. J. et al. A hemolytic transfusion reaction due to DEA 4 alloantibodies in a dog. Journal of Veterinary Internal Medicine, v. 17, n. 6, p. 931-933, dez. 2003

MESA-SANCHEZ, I. et al. Prevalence of dog erythrocyte antigen 1.1 in galgos (Spanish greyhounds). Veterinary Record, v. 174, n. 14, p. 351-351, abr. 2014.

NOVAIS, A. A.; SANTANA, A. E.; VICENTIN, L. A. Prevalence of DEA 1 canine blood group system in dogs (Canis familiaris, Linnaeus, 1758) reared in Brazil. Brazilian Journal of Veterinary Research and Animal Science, v. 36, n. 1, p. 0-0, 1999.

ODUNAYO, A. et al. Incidence of incompatible crossmatch results in dogs admitted to a veterinary teaching hospital with no history of prior red blood cell transfusion. Journal of the American Veterinary Medical Association, v. 250, n. 3, p. 303-308, fev. 2017.

OGNEAN, L. Testing of some canine blood types in transfusion compatibility assessment. Blood, 2013.

POLAK, K. et al. Dog erythrocyte antigen 1 (DEA 1): Mode of inheritance and initial characterization. Veterinary clinical pathology / American Society for Veterinary 
Clinical Pathology, v. 44, n. 3, p. 369-379, set. 2015.

RIOND, B. et al. Prevalence of dog erythrocyte antigen 1.1 in dogs in Switzerland evaluated with the gel column technique. Schweizer Archiv Fur Tierheilkunde, v. 153, n. 8, p. 369-374, ago. 2011.

SPADA, E. et al. Prevalence of dog erythrocyte antigens 1, 4, and 7 in Podenco Ibicenco (Ibizan Hounds) from Ibiza Island. Veterinary Medicine International, v. 2016, p. 1048257, 2016a.

SPADA, E. et al. Prevalence of naturally occurring antibodies against dog erythrocyte antigen 7 in a population of dog erythrocyte antigen 7-negative dogs from Spain and Italy. American Journal of Veterinary Research, v. 77, n. 8, p. 877-881, ago. 2016 b.

SPADA, E. et al. Dog erythrocyte antigens (DEA) 1, 4, 7 and suspected naturally occurring anti-DEA 7 antibodies in Italian Corso dogs. The Veterinary Journal, v. 222, p. $17-$ 21, abr. 2017.

SYMONS, M.; BELL, K. Expansion of the canine A blood group system. Animal Genetics, v. 22, n. 3, p. 227-235, 1991.

SYMONS, M.; BELL, K. Canine blood groups: description of 20 specificities. Animal Genetics, v. 23, n. 6, p. 509$515,1992$.

TOCCI, L. J. Transfusion medicine in small animal practice. Veterinary Clinics of North America: Small Animal Practice, Immunology: Function, Pathology, Diagnostics, and Modulation. v. 40, n. 3, p. 485-494, maio 2010.

VAN DER MERWE, L. L.; JACOBSON, L. S.;

PRETORIUS, G. J. The breed prevalence of dog erythrocyte antigen 1.1 in the Onderstepoort area of South Africa and its significance in selection of canine blood donors. Journal of the South African Veterinary Association, v. 73, n. 2, p. 53-56, jun. 2002.

VRIESENDORP, H. M. et al. Joint report of 1st International Workshop on Canine Immunogenetics. Tissue Antigens, v. 3, n. 2, p. 145-163, 1973.

YOUNG, L. E. et al. Blood groups in dogs-their significance to the veterinarian. American Journal of Veterinary Research, v. 13, n. 47, p. 207-213, 1952. 